\title{
Salud mental en la educación superior: una mirada desde la salud pública ${ }^{\dagger}$
}

ISSN 1794-9831

E-ISSN 2322-7028

Vol. 13 No. 1

Ene - Jun 2016

Cúcuta, Colombia

Olga Mariela Mogollón-Canal*

Diana Janeth Villamizar-Carrillo**

Sandra Licette Padilla-Sarmiento***

\section{RESUMEN}

Objetivo: describir la salud mental de los estudiantes de una institución de educación superior de Norte de Santander, a través del modelo de creencias, con miras a la construcción y aporte de una universidad saludable. Materiales y Métodos: la metodología es descriptiva, de tipo trasversal, con una muestra de 836 estudiantes, quienes respondieron un cuestionario de 125 preguntas que evaluó seis dimensiones: severidad, susceptibilidad, beneficios, barreras, motivación y claves para la acción frente a la salud mental percibida. Resultados: los estudiantes, en cuanto a la vulnerabilidad frente a su salud mental, no se perciben susceptibles, razón por la cual se involucran en conductas de riesgo; sin embargo, cuentan con factores protectores enmarcadas en la motivación y las claves para la acción que les facilitan la búsqueda de apoyo social y familiar desde el nivel de prevención; una barrera percibida se encuentra en la ausencia de políticas que el contexto en el que se desenvuelven les proporciona. Conclusiones: el modelo de creencias se constituye en una alternativa investigativa para explicar la salud mental y la intervención en la misma, que permita visualizar entornos universitarios saludables. La salud mental se convierte en un factor protector del adecuado desarrollo y rendimiento académico.

PALABRASCLAVE: estudiantes, psicología, saludmental, salud pública, vulnerabilidad ${ }^{* * * *}$.
Para citar este artículo / To reference this article / Para citar este artigo

Mogollón-Canal OM, Villamizar-Carrillo DJ, Padilla-Sarmiento SL.Salud mental en la educación superior: una mirada desde la salud pública. Rev. cienc. cuidad. 2016; 13(1): 103-120.

$\dagger$ Esta socialización cuenta la experiencia investigativa de algunos resultados de un macro-proyecto sobre universidades saludables que involucra, salud mental, sexual y física, en docentes, administrativos y estudiantes, matriculado en la vice-rectoría de investigaciones, conformado por un grupo interdisciplinar de las facultades de Salud y Educación de una institución de educación superior de Norte de Santander; aquí, se retoman los resultados de estudiantes y su salud mental.
Recibido:

4 de Febrero de 2016

Aceptado por pares: 7 de Marzo de 2016

Aprobado: 6 de Abril de 2016

* Psicóloga. Doctora en Líneas Actuales de Investigación en Psicología. Docente Asociada Directora Grupo de Investigación Psicología y Sociedad. Universidad de Pamplona. Pamplona, Colombia. Correo electrónico:

olgamariela@ unipamplona.edu.co **Psicóloga Doctora en Salud, Envejecimiento y Cognición. Docente Asociada. Docente Investigadora Departamento de Psicología. Investigadora Grupo de Investigación Psicología y Sociedad. Universidad de Pamplona. Pamplona, Colombia. Correo electrónico: jacarrillo71@ unipamplona.edu.co ***Psicóloga. Magíster en Educación .Docente Investigadora Departamento de Psicología. Investigadora activa grupo Investigación Psicología y Sociedad. Universidad de Pamplona. Pamplona, Colombia. Correo electrónico: salypasa@gmail.com ****Descriptores en Ciencias de la Salud (DeCS), en la página http://decs.bvs.br/E/ DeCS2015_Alfab-S. htm de la Biblioteca virtual en salud del proyecto BIREME, de la Organización Mundial de la Salud y de la Organización Panamericana de la Salud. 


\section{Mental health in higher education: a view from public health}

\section{ABSTRACT}

Objective: to describe the mental health of students of an institution of higher education Norte de Santander, through belief model with a view to the construction and delivery of a healthy university. Materials and Methods: the methodology is of a descriptive traversal type in a sample of 836 students who answered a questionnaire of 125 questions that evaluated six dimensions: severity, susceptibility, benefits, barriers, motivation and key elements for action against perceived mental health. Results: students in terms of vulnerability to mental health are not perceived susceptible, reason for which they engage in risky behaviors; however they have protective factors framed in motivation and the key elements for the action that facilitate the search for social and family support from a preventative level; a perceived barrier is found in the absence of policies that the context in which they operate provides. Conclusions: the belief model constitutes a research alternative explanation for the mental health and intervention in it that allows visualizing healthy university environments. Mental health becomes a protective factor for proper development and academic achievement.

KEY WORDS: students, psychology, mental health, public health, vulnerability. 


\section{A saúde mental no ensino superior: uma visão de saúde pública}

\section{RESUMO}

Objetivo: descrever a saúde mental dos estudantes de uma instituição de ensino superior Norte de Santander, através de modelo de crença, com vista à construção e entrega de uma universidade saudável. Materiais e Métodos: a metodologia é descritiva tipo transversal em uma amostra de 836 estudantes que responderam a um questionário de 125 perguntas que avaliou seis dimensões: gravidade, susceptibilidade, benefícios, barreiras, motivação e chaves para a ação contra a saúde mental percebida. Resultados: os alunos em termos de vulnerabilidade à saúde mental não são percebidos suscetíveis, porque eles se envolvem em comportamentos de risco; no entanto, eles têm enquadrado fatores de proteção em motivação e as chaves para a ação que facilitar a busca de apoio social e familiar a partir do nível de prevenção; uma barreira percebida é, na ausência de políticas que o contexto em que operam fornece. Conclusões: o modelo de crença constitui uma explicação alternativa de pesquisa para a saúde mental e intervenção em que permite visualizar ambientes universitários saudáveis. A saúde mental torna-se um fator de proteção para o desenvolvimento adequado e desempenho acadêmico.

PALAVRAS-CHAVE: estudantes, psicologia, saúdemental, saúde pública, vulnerabilidade. 
E-ISSN 2322-7028

Vol. 13 No. 1

Ene - Jun 2016

Cúcuta, Colombia

\section{INTRODUCCIÓN}

$\mathrm{L}$

a salud mental como eje y dimensión fundamental de la salud integral ha sido definida hace varios años atrás, por múltiples autores y desde diferentes enfoques, realizando énfasis particular y específico en el ajuste y en la adaptación y haciendo complejo la utilización de uno solo de ellos. La salud mental es un estado de ajuste favorable, que presupone características mentales personales particularmente deseables (1).

Es un estado de bienestar que posibilita al individuo la consciencia plena de sus potencialidades y de sus capacidades para afrontar las situaciones de tensión que el día a día de la vida le presenta; destaca también la habilidad para trabajar de forma productiva y fructífera, contribuyendo con esto a la comunidad de la cual hace parte (2).

La salud mental posibilita el desarrollo eficaz de todas las áreas en las que el individuo se moviliza y asegura el desarrollo del ecosistema. Hace referencia también a la calidad de vida y al bienestar que el sujeto percibe y le proporciona su día a día; aquí intervienen sus creencias, sus estilos de vida, sus recursos y sus potenciales.

La salud mental positiva se define como una forma óptima de funcionamiento psicológico y una sensación general de bienestar. En cambio, la salud mental negativa incluye facetas perjudiciales como problemas de salud, psicopatología o trastornos psiquiátricos (3).

Así, el concepto de salud mental se entiende como un proceso cíclico y activo de ajuste constante, tanto de las necesidades internas como de las que el medio físico y social establece.

En Colombia se han consolidado, hasta el momento, cuatro (4) encuestas nacionales de salud mental; la primera, desarrollada en 1993, que indagó por los procesos comunicativos familiares, el establecimiento de las redes de apoyo social, los trastornos de ansiedad y depresión, el alcoholismo y los factores psicosociales, entre otros.

La segunda, implementada en 1997, se apoyó en la entrevista diagnóstica compuesta, generada por la
Organización Mundial de la Salud (OMS) y el Manual Diagnóstico y Estadístico de los Trastornos Mentales (DSM IV) y abordó a 15.408 personas.

La tercera, desarrollada en el año 2003 como parte de la Encuesta Mundial de Salud Mental realizada por la OMS con una cobertura de 4.426 personas en un rango de edad de los 18 a los 65 años, utilizó nuevamente la entrevista diagnóstica compuesta, versión computarizada; valorando las situaciones de discapacidad y las condiciones crónicas.

La cuarta y última, generada durante el año 2015, visibiliza por primera ocasión aquellos problemas mentales que son objeto de atención social y clínica, pero aún no representan una enfermedad o trastorno mental diagnosticado y, además, aumenta el rango de edad, incluyendo el diagnóstico de niños y niñas de 7 años.

Alrededor de 400 millones de personas en el mundo presentan o han presentado alguna vez en su vida situaciones asociadas a los trastornos de salud mental (4). Es necesario comprender que la magnitud y el impacto que estas personas suponen, no se corresponde con los recursos que se dedican a su investigación y atención (5).

Debe considerarse que los trastornos mentales, como cualquier otro motivo de consulta en relación con la salud mental, aparecen a edades más tempranas que otras condiciones médicas de tipo crónico y no transmisible y, por ende, la carga de enfermedad atribuible a este tipo de eventos es considerable y requiere de acciones prioritarias de promoción y prevención (4).

Históricamente, desde 1985, con la publicación de la carta de Ottawa por parte de la OMS (6), en el tema de la promoción de la salud despertó el interés por trabajar en investigaciones que aporten en el campo del cómo se pueden acompañar las movilizaciones del comportamiento hacia estilos de vida saludables.

Las investigaciones sobre universidades como entornos saludables que permiten el desarrollo de determinantes personales cuentan con dos desarrollos de interés para esta investigación: el primero corresponde a estudios centrados en la valoración de las percepciones y vivencias de los propios 
estudiantes universitarios y los agentes gestores; el segundo concierne a investigaciones enfocadas en los contextos universitarios que ayudan a la creación de estrategias, espacios y posibilidades a través de planes, programas y proyectos para que los estudiantes posibiliten su salud integral de forma positiva y de acuerdo a su ciclo vital, simultáneamente con su desarrollo académico.

En cuanto a las vivencias de los propios estudiantes, algunos de los factores de riesgo a los que se ven expuestos y que cobran especial importancia en esta etapa, se enmarcan dentro de prácticas sexuales sin protección, sedentarismo y malos hábitos de alimentación, entre otros, todos vinculados a estilos de vida (7).

Investigaciones españolas (8) concluyen que los estudiantes universitarios se ponen en riesgo, con facilidad, en temas de salud mental como el consumo de sustancias psicoactivas, directamente relacionadas con dificultades en las necesidades académicas, familiares y el manejo del círculo de los pares.

Un ejemplo, enfocado en los contextos universitarios, es la experiencia de una universidad colombiana en el eje cafetero que desarrolló estudios enfocados a encontrar alternativas de intervención con universitarios, convocando a profesionales de educación y salud y haciendo alianzas externas que permitieron fomentar estilos de vida saludables (9).

Otra experiencia la integra el movimiento de universidades por la salud, constituido como un proceso que impulsa el fortalecimiento de la salud escolar. Aquí se considera el bienestar universitario como el eje transversal que media todos los procesos de la vida universitaria y promueve el desarrollo y la satisfacción de todos sus actores (10).

La investigación que este artículo presenta se realiza en una universidad nortesantandereana, entendiendo la salud mental como un estado continuo que permite el reconocimiento de los potenciales individuales $\mathrm{y}$, a partir de ellos, el afrontamiento diario de los diferentes estresores que cada una de las situaciones universitarias presenta.

La salud mental, para las personas que integran esta universidad, se constituye en un elemento fundamental y una inversión con alta rentabilidad que incluye dimensiones personales y sociales.

De acuerdo con esta investigación y con el modelo de creencias utilizado para la misma, los actores educativos conformados por estudiantes presentan características particulares que permiten establecer puntos de partida y realizar análisis a partir de ellos. A continuación se encuentran los principales elementos de análisis en torno a la salud mental.

Se retoma el modelo de creencias de salud, el cual intenta describir y predecir las conductas que una persona realizará si siente que con esto puede evitar un riesgo y cree que puede realizar la acción (11).

Este modelo ha sido uno de los utilizados en los diseños de investigación relacionados con los factores cognitivos que influyen en la adopción de conductas preventivas ante enfermedades $y / 0$ situaciones crónicas, puesto que propone que dichos factores cognitivos pueden o no favorecer la probabilidad de ocurrencia de una acción y/o conducta preventiva (12).

Se centra en la explicación y predicción del comportamiento preventivo en salud, tomando como base las interacciones de las creencias que tienen las personas. El modelo se extiende a las reacciones que las personas tienen frente a los síntomas de una enfermedad y/o situación y a su comportamiento en general diagnosticado, dando lugar a la adherencia al tratamiento (13).

El modelo de creencias en salud toma como referente teórico el trabajo referido a la motivación y a la percepción; a la toma de decisiones que las personas realizan frente a las situaciones de elección de alternativas, de opción comportamental con implicaciones en la salud; a la solución de problemas y a las prácticas de la salud pública (13). Pretende centrarse en las percepciones de amenazas y en los valores y creencias que permiten aumentar o disminuir dichas amenazas, lo cual está directamente relacionado con los niveles de vulnerabilidad y la severidad percibida del riesgo (14).

Específicamente, la probabilidad de que una persona adopte una conducta para prevenir una enfermedad dependerá de: (a) grado de motivación, (b) auto percepción como potencialmente vulnerable ante 
la enfermedad, (c) percibir la condición como amenazante, (d) estar convencido de que la intervención es eficaz y (e) percepción de poca dificultad en la conducta de salud (15).

La susceptibilidad hace alusión a la opinión personal $\mathrm{y} / \mathrm{o}$ percepción subjetiva que una persona puede experimentar frente al riesgo o a la vulnerabilidad que tiene de contraer y/o padecer una determinada condición de salud, enfermedad u otros acontecimientos vitales, aspectos que repercuten en la adhesión al tratamiento.

De esta forma, si la persona minimiza el riesgo propio, es decir, considera que tiene poca probabilidad de que un suceso le ocurra, es más probable que no adopte las recomendaciones que los profesionales de la salud establezcan. Contrario a esto, una susceptibilidad alta permite que el interés por el aprendizaje y desarrollo de conductas saludables aumente (16).

La edad y la experiencia propia son determinantes en la vulnerabilidad percibida (16). La edad, por ejemplo, puede llevar a muchas personas a creer que los acontecimientos negativos sólo les suceden a los demás, aumentando la ausencia de conductas preventivas. La experiencia previa incluye la experiencia personal y la experiencia vicaria que amigos y familiares puedan tener.

La severidad percibida se refiere a la opinión que la persona tiene sobre la gravedad de una afección y las secuelas que ésta puede generar(17). Hace alusión a la probabilidad de verse afectado por la enfermedad o de no recibir tratamiento; se revisa la gravedad clínica, impacto sobre la propia apariencia, efectos sobre el trabajo, la vida familiar y las relaciones sociales (11).

Los beneficios percibidos se constituyen en la estimación que el individuo tiene de los aportes que obtendría realizando la acción saludable frente a los costes o barreras que se impliquen en ellos; la dirección de la acción se ve influenciada por la efectividad que el sujeto considera tendrá la acción empleada para minimizar la amenaza (11).

Las barreras percibidas se refieren a los costos tangibles y psicológicos que la acción sugerida puede presentar en virtud de la oposición a la ejecución de la conducta en cuestión. Así, un individuo puede considerar un determinado curso de acción como realmente efectivo para enfrentarse a un trastorno de salud pero, al mismo tiempo, puede verlo como costoso, desagradable o dolorosos (18).

Las claves para la acción se constituyen en aquellas estrategias que permiten que el sujeto comprenda la forma en la que debe desarrollar las acciones, el curso, el procedimiento y/o el camino que debe recorrer para implementar y desarrollar las conductas y/o comportamientos saludables a partir de la toma de decisiones (18).

En el ámbito de la salud, estas claves pueden ser internas o externas. La intensidad necesaria de una de estas claves para desencadenar la conducta puede variar de sujeto a sujeto, e incluso en el mismo sujeto dependiendo de sus niveles de susceptibilidad y del grado de severidad percibido (18).

La Motivación hacia la Salud es una dimensión importante para el inicio, desarrollo y mantenimiento de conductas y acciones saludables. En ella tienen lugar procesos cognitivos y psicológicos que determinan, en una situación específica, la fuerza y la dirección de las acciones (11).

\section{MATERIALES Y MÉTODOS}

La metodología utilizada fue descriptiva, exploratoria y transversal. Los participantes pertenecían a todas las facultades de la institución de educación superior, a quienes se les abordó en salones de clase y espacios al aire libre, teniendo en cuenta la aprobación del Comité de Ética de la Universidad y aplicándose un consentimiento informado, de acuerdo con las normas de la Asociación Colombiana de Facultades de Psicología (ASCOFAPSI).

El tipo de muestreo en voluntario es intencional, el cual sobrepasó la formula estipulada.

Para el tamaño de la muestra se utilizó la siguiente fórmula:

$$
n=\frac{N z^{2} p q}{(N-1) E^{2}+z^{2} p q}
$$

En donde:

$\mathrm{N}$ : Número total de estudiantes presenciales en la 
sede central de la Universidad ( $\mathrm{N}=8360$ en el segundo semestre de 2013)

p: Probabilidad de elegir un estudiante aleatoriamente $(\mathrm{p}=0,5)$

$\mathrm{q}$ : Probabilidad complementaria a $\mathrm{p}(\mathrm{q}=1-\mathrm{p} ; \mathrm{q}=1-$ $0,5=0,5)$

E: Error de estimación $(E=6,24 \%)$

z: Depende del coeficiente de confianza (95\%); $(\mathrm{z}=1,96)$.

Al sustituir los valores anteriores en la expresión para el tamaño de muestra se obtiene que ésta debe ser como mínimo de 368 estudiantes para el caso de la investigación, la cual contó con 836 participantes voluntarios o intencionales. Esto minimizó el error de estimación y confirió a los resultados una mayor representación. La muestra correspondió a un $75 \%$ de adultos jóvenes, un $18 \%$ de adolescentes y un aproximado de $7 \%$ de adultos medios, de diversas procedencias.

El instrumento utilizado fue el Cuestionario de Evaluación Integral de la salud mental para estudiantes, el cual fue creado para población estudiantil y constó de un aproximado de 206 preguntas. La validez y la confiabilidad del instrumento se obtuvieron a través de 5 evaluaciones de juicio de expertos y 9 pruebas piloto, las cuales se distribuyeron en tres momentos hasta finalizar con la consolidación del instrumento utilizado. Los resultados obtenidos con la aplicación de la prueba piloto permitieron ajustar el lenguaje del instrumento y la compilación de las categorías de evaluación en un número menor de reactivos, quedando finalmente una prueba con 125 ítems. Su aplicación fue manual, en espacios individuales y colectivos.

El instrumento contó con varias escalas de valoración. Algunas preguntas fueron realizadas de forma abierta y recibieron un análisis categorial, con sus respectivas unidades de análisis. Estas clasificaciones fueron avaladas por tres investigadores, de forma particular, hasta llegar a la clasificación final de dichas categorías. Otras preguntas se construyeron mediante una escala tipo Likert, con 5 opciones de respuesta enfocadas a dos de carácter favorable, dos de carácter desfavorable y una neutral.

Metodológicamente, se llevó en cada proceso diario de campo y registros, los cuales se diligenciaron de forma escrita con cada uno de los participantes e inmediatamente, de forma verbal, se leyeron las instrucciones de los mismos, donde se especificaba que el estudio era voluntario, que los resultados eran de carácter general y no particular, sólo con fines investigativos y explicando el objetivo de la investigación.

La información recolectada se analizó a través del paquete estadístico SPSS versión 20 y, de acuerdo con estas clasificaciones, se realizó una descripción porcentual de cada una de las dimensiones, teniendo en cuenta el modelo de creencias en salud junto con una descripción narrativa e interpretativa de las diferentes tablas que permitieron el diagnóstico inicial.

Los resultados hacen parte de un macroproyecto de investigación que abordó también población docente y administrativa de una institución de educación superior; que contó con el aval institucional a través de la Vicerrectoría de Investigaciones, la cual autorizó el permiso para su desarrollo. Los propósitos y la metodología a utilizar cuentan con el aval dado por el Comité de Ética e Impacto Ambiental de la institución, con acta 004 del 24 de septiembre del año 2013.

\section{OBJETIVOS}

\section{Objetivo general}

Describir la salud mental de los estudiantes de una institución de educación superior de Norte de Santander, utilizando las dimensiones del modelo de creencias en salud, que permita, a futuro, la construcción de políticas encaminadas a la consolidación de una universidad saludable.

\section{Objetivos específicos}

- Analizar el grado de severidad y susceptibilidad percibida sobre los aspectos de la salud mental de los estudiantes, desde el modelo de creencias.

- Determinar los beneficios y barreras percibidos por los estudiantes para su salud mental, desde el modelo de creencias.

E-ISSN 2322-7028

Vol. 13 No. 1

Ene - Jun 2016

ia




\section{RESULTADOS}

La recolección de los datos se realizó durante el año 2013; las limitaciones estuvieron enfocadas en la temática y su intimidad, al ser un tema de difícil acceso y que representa, en ciertos sujetos, confrontación con las realidades propias del ser humano.

La muestra representativa contó con un 42,2\% de hombres y un $57,8 \%$ de mujeres; el $75 \%$ estuvo representado en adultos jóvenes, $18.2 \%$ adolescentes y el $6.8 \%$ adultos medios. El estado civil del 86,4 $\%$ de los encuestados fue soltero, el $8.3 \%$ convivía con su pareja y el $1.4 \%$ era casado. La religión predominante fue la católica $(69,6 \%)$, seguida del cristianismo (15.6\%). El $6.5 \%$ refirió no tener religión, el $3.5 \%$ no sabe no responde y los demás de diferentes religiones. El $30 \%$ de los estudiantes manifestó que vivía con su familia, el $20.2 \%$ vivía solo él con compañeros de estudio, el $9.1 \%$ con personas de su lugar de origen, el $7.7 \%$ con otros familiares y el $7.3 \%$ con su pareja.
Respecto a su nivel socioeconómico, el 36,4 \% pertenecía al estrato dos, el 35,6 \% manifestó pertenecer al estrato uno, el $22,8 \%$ al estrato tres, el $4.7 \%$ al estrato cuatro y menos del $1 \%$ al estrato cinco y seis.

\section{Severidad percibida}

En cuanto a la salud mental de los estudiantes de la institución de educación superior, en la categoría de severidad percibida se observa que los problemas psicológicos afectan bastante la vida cotidiana de los estudiantes $(46,7 \%)$ y mucho $(33 \%)$. Lo que más afecta su salud mental es la familia, en primer lugar, y la vida académica, en segundo aspecto; un porcentaje medio mencionó que ninguna situación le afecta y un pequeño porcentaje opinó que su relación de pareja y los amigos.

A continuación se presenta la Figura 1, que contiene los resultados obtenidos respecto a la dimensión severidad percibida por los estudiantes en cuanto a su salud mental.

Figura 1. Análisis parcial dimensión severidad percibida estudiantes universitarios.

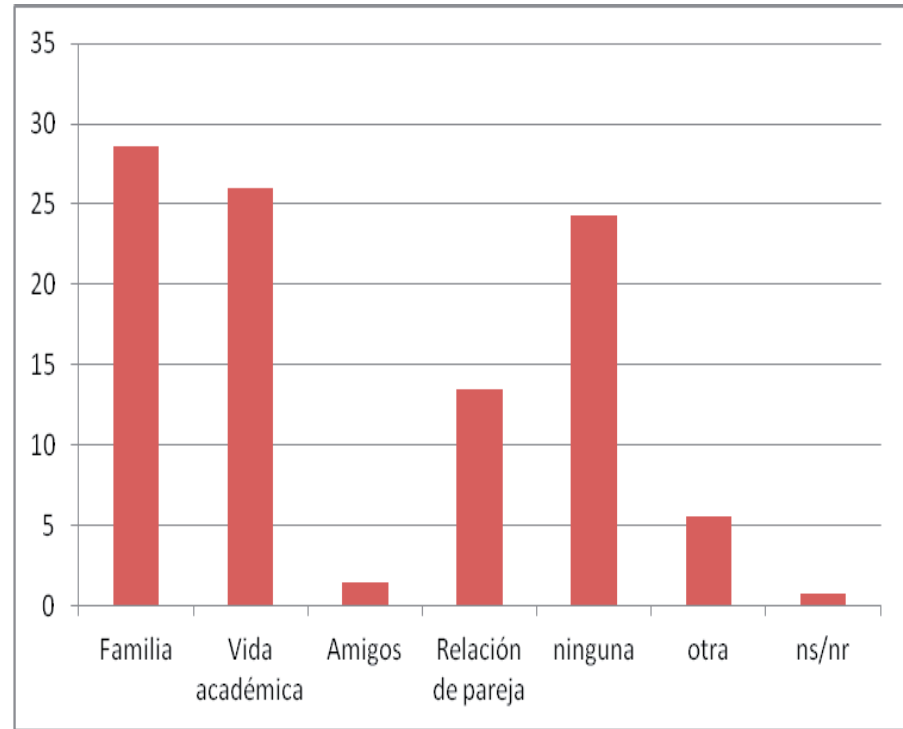

Fuente: Encuesta estudiantes evaluación integral de la salud mental, 2013.

Cuando los estudiantes ven afectada su salud mental, el $44.5 \%$ considera que algunas veces ha perdido el control en ciertas situaciones, seguido de un $23.8 \%$ que considera que casi nunca.
Es de resaltar que existe un número de estudiantes que reportó que casi siempre pierde el control, aunque no llega a ser el $13 \%$, acompañado de un 4.9 $\%$ que refirió que siempre pierde el control en ciertas 
situaciones, ya que estos dos últimos, en frecuencia, dan un significante considerable.

La mayoría de los estudiantes $(67,2 \%)$ está totalmente de acuerdo en que, al consumir sustancias psicoactivas, se puede generar adicción a ellas. Un aproximado de $30 \%$ de estudiantes no percibe la severidad de que el consumir sustancias psicoactivas puede generar adicción.

Respecto al deterioro físico producto de un problema de salud mental, la mayoría se encuentra enmarcada en que nunca se les ha presentado deterioros físicos que sean producto de un problema de salud mental. Sin embargo, algunos reconocen haber presentado esta situación.

\section{Susceptibilidad percibida}

A este respecto, los estudiantes perciben que su estado de salud mental es el resultado de la estabilidad afectivo-emocional $(39,1 \%)$, los hábitos $(26,1 \%)$ y su autoestima $(21,3 \%)$. En una menor medida se encuentran los aspectos del estado físico $(6,1 \%)$ y la economía $(4,7 \%)$.

Igualmente, perciben que la frecuencia con la que se ha presentado una situación que afecte su salud mental es: nunca la ha presentado $(37,7 \%)$, algunas veces se ha presentado $(32,1 \%)$, seguido del casi nunca $(21,9 \%)$. Es de resaltar que el $8 \%$ opina que casi siempre o siempre la presenta.

Respecto a la posibilidad de presentar un problema de salud mental, la mayoría de la población estudiantil
(72\%) considera que es muy baja o baja. Un porcentaje significativo de estudiantes percibe vulnerabilidad de presentar un problema de salud mental en las opciones muy alta, alta y medianamente $(24,1 \%)$, quedando un $4 \%$ que no sabe o no responde.

Al preguntar a los estudiantes si el estrés afecta su salud mental, la mayoría de las respuestas se encuentra en las opciones siempre, casi siempre y algunas veces $(78,5 \%)$. Una minoría no considera que el estrés afecte su salud mental, junto con no sabe o no responde con un $21,5 \%$.

La percepción individual de su estado de salud mental se encuentra entre satisfactorio y muy satisfactorio. Sin embargo, es necesario tener en cuenta que hay un promedio de $11 \%$ de estudiantes que no considera que su estado de salud mental sea satisfactorio.

Los estudiantes perciben que pueden estar en riesgo respecto de su salud mental, por lo que se consideran vulnerables a situaciones específicas como el estrés, las dificultades afectivo-emocionales, los malos hábitos y el manejo de su autoestima.

Aunque se creen vulnerables, el reconocimiento de presentar situaciones que no les permitan una buena salud mental es bajo, lo que es coherente con sus altos niveles de satisfacción respecto a su actual estado de salud mental.

La Figura 2 presenta los resultados parciales obtenidos en la dimensión susceptibilidad percibida por los estudiantes en cuanto a su salud mental. 
Figura 2. Análisis parcial dimensión susceptibilidad percibida estudiantes universitarios.

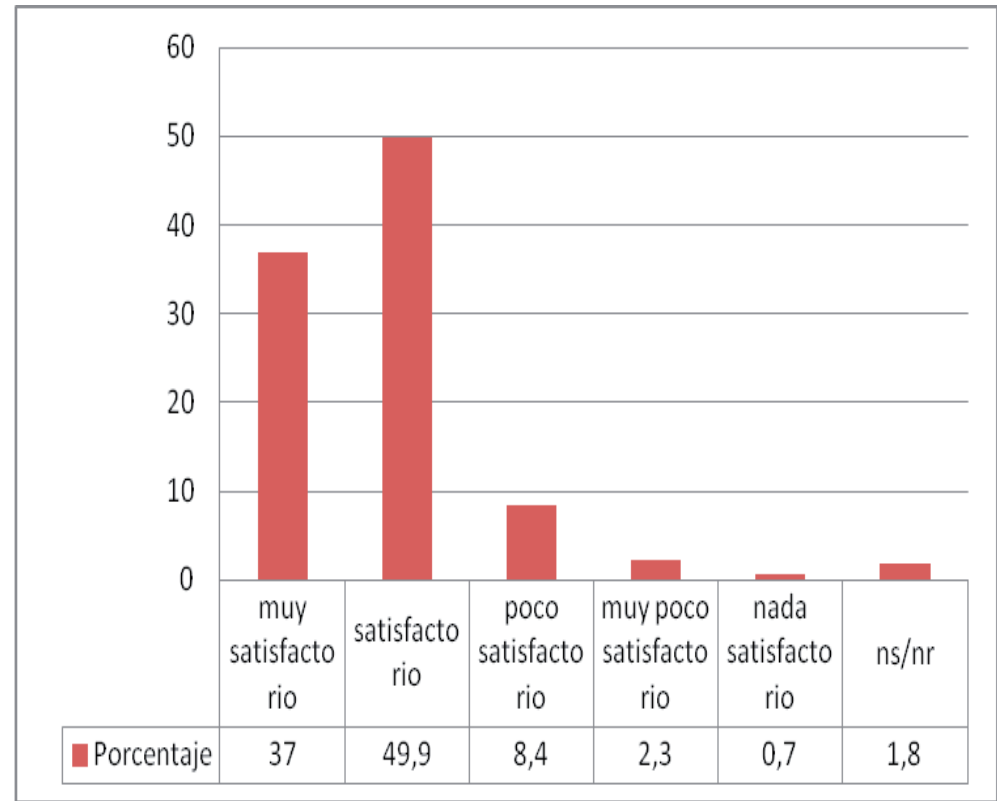

Fuente: Encuesta estudiantes evaluación integral de la salud mental, 2013

\section{Beneficios percibidos}

Con relación a los beneficios percibidos, el 33.6\% de los estudiantes cree en el apoyo que le pueda dar un profesional de la salud mental y si presentara una situación de amenaza por un trastorno de salud mental seguiría, en su mayoría, las recomendaciones que el psicólogo hiciera, percibiendo como beneficioso seguir un tratamiento cuando se requiere.

Sin embargo, existe una frecuencia de estudiantes que considera que este apoyo no es necesario. La gran mayoría percibe que las relaciones entre un profesional de la salud mental y el consultante deben ser adecuadas.

Los estudiantes interpretan que siempre cuentan con apoyo al tener un problema psicológico. La familia se considera como el principal apoyo emocional al presentarse un problema psicológico $(82,9 \%)$, seguida de los amigos (10,2\%). Al mismo tiempo, consideran que si presentara alguna enfermedad psicológica, se la tratarían.

E174.4\% de los estudiantes no consume medicamentos sin prescripción médica y considera que el consumo de sustancias como cafeína, cigarrillos, alcohol, marihuana, cocaína y otras no ayuda a disminuir la ansiedad. Sin embargo, es importante atender el porcentaje de la población que sí lo hace y sí lo percibe.

Las creencias de los estudiantes en cuanto a la efectividad de los servicios psicológicos poseen un alto porcentaje de favorabilidad, puesto que reconocen el beneficio de seguir sus acciones y sus recomendaciones.

No obstante, es necesario atender a la población que, aunque es mínima, no lo considera necesario, además de seguir atendiendo a la población que sí lo considera, ya que casi un $16 \%$ de los estudiantes reporta el consumo de medicamentos sin prescripción médica y el $22 \%$ considera que el consumo de sustancias psicoactivas ayuda a disminuir la ansiedad.

De forma gráfica se muestran los beneficios percibidos por los estudiantes en cuanto a su salud mental, en la Figura 3. 
Figura 3. Análisis parcial dimensión beneficios percibidas estudiantes universitarios.

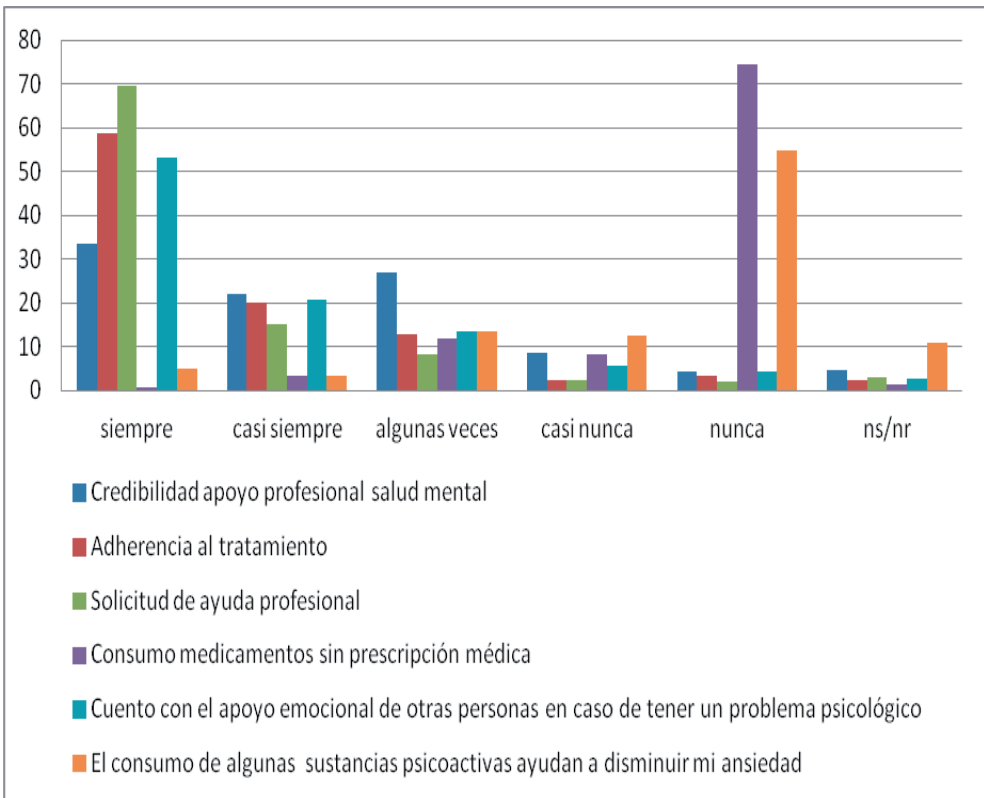

Fuente: Encuesta estudiantes evaluación integral de la salud mental, 2013.

\section{Barreras percibidas}

Dentro de las barreras percibidas por los estudiantes para su salud mental, existen diversidad de opiniones frente a la facilidad para acceder a un profesional de la salud mental: el porcentaje del $28.5 \%$ se encuentra en la opción de algunas veces. Esto puede ser analizado desde variables como la Entidad Promotora de Salud (EPS) de afiliación, la facilidad que brinda para la consulta y el contexto cultural, entre otros aspectos. Casi un $30 \%$ considera que nunca o casi nunca tiene facilidad de acceder a un profesional de la salud mental, percibiendo una barrera. Si bien es cierto que los estudiantes consideran la necesidad de acudir al psicólogo, el poder hacerlo se convierte en la barrera más importante.

Respecto al afrontamiento de las situaciones difíciles a nivel personal, el $49.2 \%$ y el $34.6 \%$ de la población se enmarca en el siempre y el casi siempre. Frente a las barreras percibidas es claro que tienen mayor peso las externas y contextuales que las internas e individuales, lo cual resulta coherente con la susceptibilidad percibida.

Las opiniones están divididas entre los obstáculos percibidos para el mantenimiento de estilos de vida saludable frente a la salud mental. La frecuencia del $29.4 \%$ y el $25.4 \%$ se encuentra en el nunca y casi nunca perciben obstáculos, seguido del algunas veces, casi siempre y siempre, lo cual hace necesaria una intervención integral desde la psicología de la salud para el manejo de estos obstáculos. La Figura 4 muestra las barreras percibidas por los estudiantes en cuanto a su salud mental. 
Figura 4. Análisis parcial dimensión barreras percibidas estudiantes universitarios.

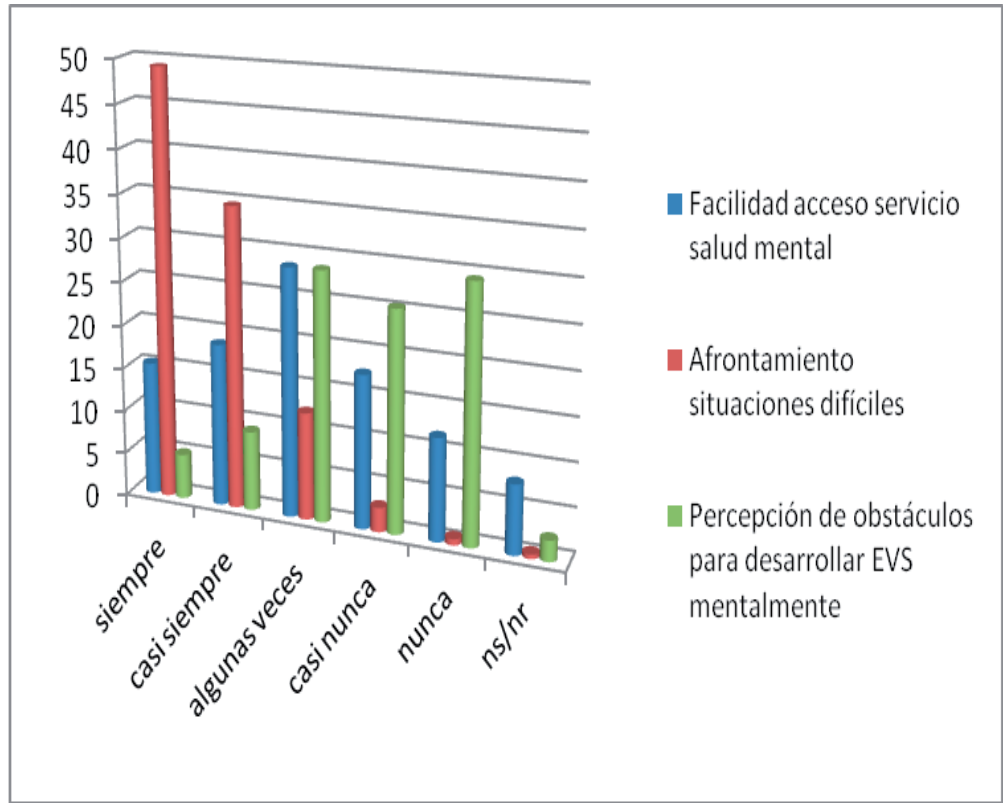

Fuente: Encuesta estudiantes evaluación integral de la salud mental, 2013.

\section{Motivación para la salud}

En cuanto a la motivación para la salud, los estudiantes perciben, en un $38.9 \%$, que tienen la capacidad para disminuir los riesgos de tener dificultades psicológicas, asumiendo comportamientos saludables, para lo cual realizan actividades de disfrute y bienestar, seguidas de actividades de autoestima y fortalecimiento de la comunicación, actividades para acrecentar la actividad afectivo-emocional y para la toma de decisiones, entre otras.

Existe un porcentaje de estudiantes del $21.6 \%$ que percibe que casi nunca o nunca tiene la capacidad para disminuir el riesgo de dificultades psicológicas asumiendo comportamientos saludables.

Dentro de los procesos autorregulatorios de la motivación intrínseca de los estudiantes se encuentra la capacidad de poder disminuir el riesgo a través de comportamientos saludables, con elementos que incluyen el balance y potencial de la salud, encontrándose actividades internas y externas para el desarrollo de estilos de vida saludables.

\section{Claves para la acción}

En la categoría de claves para la acción, al indagar por la posibilidad de utilizar estrategias (notas, alarmas, una persona cercana) para recordar la cita psicológica en el momento de acudir al psicólogo, las percepciones de los estudiantes se encuentran en un porcentaje similar entre no hacerlo y hacerlo (19.4\% algunas veces, $15.8 \%$ siempre y $14.4 \%$ casi siempre.)

Por otra parte, existe un porcentaje de estudiantes $(8,4$ $\%$ ) que no sólo se perciben vulnerables, sino que se perciben con una salud mental poco satisfactoria y no adecuada, aspecto que debe ser tenido en cuenta para el diseño y ejecución de un programa de intervención.

Consideran también, en un porcentaje del 37.1 $\%$, que los medios de comunicación ayudan en el proceso de prevención de los problemas que a nivel de salud mental pueden presentarse, encontrando en ellos claves externas que pueden ser herramientas imprescindibles para la salud mental.

Es importante tener en cuenta el porcentaje 
aproximado de $37 \%$ de estudiantes que percibe que los medios de comunicación casi nunca y nunca ayudan en el proceso de la prevención, con el propósito de establecer acciones que permitan la consolidación de estas estrategias como positivas y/o la necesidad de utilizar otro tipo de claves para empoderar a las personas en el proceso de prevención de todo aquello que afecte su salud mental.

Es relevante mencionar que, en su gran mayoría (70.1
$\%)$, los estudiantes perciben que el ambiente donde se desenvuelven de forma general es una clave esencial que les permite mantener una salud mental adecuada. Sin embargo, un porcentaje menor considera que el ambiente actual no les permite mantener una salud mental adecuada.

Los resultados parciales de la dimensión claves para la acción de los estudiantes de la muestra respecto su salud mental, se pueden apreciar en la Figura 5.

Figura 5. Análisis parcial dimensión claves para la acción estudiantes universitarios.

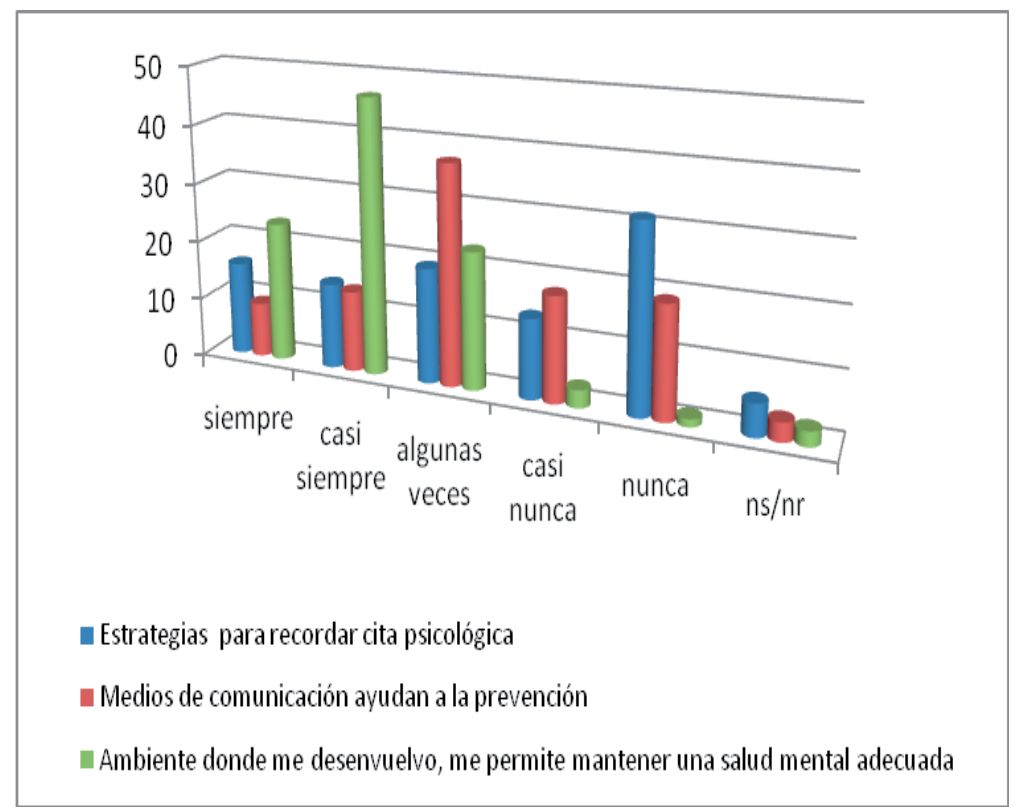

Fuente: Encuesta estudiantes evaluación integral de la salud mental, 2013.

\section{DISCUSIÓN}

La salud mental de los estudiantes universitarios es la base fundamental para su rendimiento académico $\mathrm{y}$, por tanto, debe ser factor clave en las políticas y lineamientos de las universidades saludables (15).

El número de jóvenes estudiantes de educación superior con trastornos mentales ha ido aumentando progresivamente y presenta graves problemas psicológicos, como lo demuestra el reporte de estudiantes que buscan ayuda en los servicios que ofrecen las instituciones (19).

Fue significativo el descubrir cómo el modelo de creencias, desde sus diferentes referentes teóricos, es una herramienta fundamental que ayuda a la caracterización de la salud mental en estudiantes universitarios.

Existe un trabajo implícito que se llevó a cabo en esta investigación, que cabe resaltar y tiene que ver con la interdisciplinariedad del equipo general de investigación en el Grupo de Investigación Psicología y Sociedad, dando una mirada desde diferentes disciplinas de la salud y la educación, aspecto relevante en el trabajo en salud pública.

El quehacer desde la psicología de la salud debe cuestionarse hacia la necesidad de abordar las percepciones respecto a la gravedad que el sujeto pueda tener del estado de salud. Es de resaltar que en esta muestra poblacional se evidencio un porcentaje $(29.4 \%)$ de estudiantes que no percibe ninguna 
E-ISSN 2322-7028

Vol. 13 No. 1

Ene - Jun 2016

Cúcuta, Colombia afectación frente a las situaciones del contexto respecto a su salud mental.

Es fundamental trabajar desde movilizaciones y sensibilizaciones, puesto que los resultados apuntan a la necesidad de desarrollar programas de promoción, mantenimiento y acrecentamiento de la salud mental, teniendo en cuenta los altos porcentajes de estudiantes que sí perciben la afectación de ésta, lo que prevé una necesidad de acompañamiento psicológico constante.

Por otra parte, sí existe una porción de estudiantes $(11.4 \%)$ que no sólo se percibe vulnerable, sino que se percibe con una salud mental poco satisfactoria y no adecuada, aspecto que debe ser tenido en cuenta para el diseño y ejecución de un programa de intervención. Este factor apoya lo encontrado en la Encuesta Nacional de Salud Mental 2015 (ENSM), respecto a los problemas mentales de la población colombiana, donde se recomienda visibilizar los problemas mentales como prioritarios, fortalecer las capacidades para detección de problemas mentales en los entornos en niños (problemas de aprendizaje), adolescentes (ansiedad, dificultades en el hábito de comer) y adultos (consecuencias psicológicas de eventos traumáticos), y remisión a los servicios de salud (20).

Es relevante el porcentaje de estudiantes que considera que las sustancias psicoactivas ayudan a disminuir la ansiedad; si bien no es un porcentaje grande, sí se debe tener en cuenta para la prevención y manejo del consumo.

Un aproximado del $30 \%$ de estudiantes no percibe la severidad de que, al consumir sustancias psicoactivas, se genere adicción. Esto puede significar un alto riesgo para el consumo de las mismas, para lo cual es recomendable tener en cuenta lo afirmado por la ENSM (2015) sobre la necesidad de trabajar en la detección temprana y atención oportuna de consumo de alcohol y otras drogas (20).

También se evidenció un porcentaje de estudiantes $(39.1 \%)$ que considera que el ambiente actual no le permite mantener una salud mental adecuada, situación importante de abordar desde las áreas de la salud pública, con el propósito de ajustar el medio a las necesidades de la población.

Lo anterior refuerza lo contenido en el nuevo concepto de salud, donde se destaca que no solamente son los comportamientos que las personas realizan los promotores de la enfermedad, sino que pone el acento, además, en el ambiente (21).

Los resultados aquí encontrados son paralelos a investigaciones (22) que establecen la relevancia y la afectación del estrés en los jóvenes universitarios, debido a la responsabilidad que tienen sobre su salud, la vida académica y la situación financiera. Por lo tanto, los años de la universidad se aceptan como unos de los más estresantes al relacionar rendimiento académico, salud mental y desarrollo profesional (22).

De esta forma, las instituciones de educación superior suelen ser consideradas como espacios de encuentro que generan niveles altos de estrés, puesto que los estudiantes deben enfrentarse, de forma cotidiana, con una serie de demandas que, cognitivamente hablando, consideran como estresantes y que al ser prolongadas y constantes pueden convertirse en enfermedades de salud mental (23).

Los resultados encontrados en este estudio apoyan otras investigaciones recientes como la $\operatorname{ENSM}(4,20)$ sobre la necesidad de planificar estudios nacionales de prevalencia y una juiciosa revisión de las políticas en salud pública, haciendo necesaria la inclusión de eventos en salud mental como de intervención prioritaria.

Desde las instituciones de educación superior, la responsabilidad sobre la salud mental de los estudiantes es factor fundamental para el diseño y manejo de estas políticas que redundarán no sólo en estudiantes comprometidos con su carrera, sino, más adelante, en profesionales responsables y adaptados a su medio social y laboral.

Es este un paso para la implementación de la universidad saludable (15), puesto que "el desarrollo de una cultura real a nivel universitario, exige voluntad política institucional que se traduzca en la formulación de disposiciones que garanticen sostenibilidad de los procesos, el desarrollo de estructuras y programas de Universidades Promotoras de Salud, con talento humano cualificado y recursos financieros que garanticen la sistematización y permanencia de las acciones". 
De otro lado, este estudio apoya lo afirmado acerca de que la promoción de la salud, además de lo personal y lo conductual, tiene en cuenta como estrategia más global para su aplicación y difusión cinco componentes básicos interrelacionados entre sí: la construcción de políticas públicas saludables, la creación de entornos o ambientes favorables, el fortalecimiento de la acción comunitaria y la participación social, el desarrollo de habilidades personales para el fomento de la salud y la reorientación de los servicios de salud (24).

La preocupación por la salud mental de los estudiantes universitarios se da a nivel mundial y evidencia que, a pesar de que se están empezando a entender algunas de las barreras sociales y facilitadores para ayudar a la búsqueda de las soluciones de las dificultades de salud mental entre los estudiantes universitarios, hay una sorprendente falta de pruebas sobre cómo las intervenciones a nivel de la escuela, las políticas y los recursos afectan la búsqueda de ayuda (25).

El modelo de creencias se convierte en una alternativa viable para hablar de estilos de vida saludables y conductas de prevención frente al riesgo, su dimensión motivación es fundamental para asumir y mantener conductas saludables (26) acompañadas de información previa sobre la forma de prevenir el riesgo y disminuir, de esta manera, la susceptibilidad y vulnerabilidad junto a la gravedad de las consecuencias de la conducta de riesgo.

Investigaciones paralelas concluyen, en cuanto a la susceptibilidad percibida, que la población universitaria considera mínima la posibilidad de estar en riesgo (27), convirtiéndose en una población vulnerable que asume comportamientos de riesgo con facilidad, lo cual se confirma en los resultados de esta investigación, a partir de las dos categorías del modelo de creencias de gravedad percibida y susceptibilidad percibida.

El interés por trabajar los aspectos contextuales y específicamente las políticas de las instituciones de educación superior ha ido en aumento durante las últimas dos décadas; estudios sobre salud mental y salud sexual y reproductiva aportan al desarrollo de estos entes en Norte de Santander, centrando sus esfuerzos en las actitudes sexuales de adolescentes universitarios, asunto que se ve reflejado directamente en la salud mental positiva, haciendo referencia a las actitudes, a las posturas liberales o conservadoras y a los riesgos que se asumen (28).

En la misma línea, la salud sexual aporta a la salud mental de forma integral desde el aprendizaje, frente a la comunicación y resolución de conflictos en la vida universitaria, a través del ejercicio de roles y poderes (29) que permiten el desarrollo académico integral de estudiantes universitarios.

Por tanto, la relevancia de esta investigación es fundamental para el progreso en el cubrimiento de la salud mental de los estudiantes universitarios en Colombia a futuro y aporta significativamente como un campo de acción donde el plan decenal de salud pública anuda esfuerzos en su línea de salud mental (30).

\section{CONCLUSIONES}

En cuanto a la salud mental de los estudiantes, en la categoría de severidad percibida, se observa que los problemas psicológicos afectan bastante la vida cotidiana de ellos, siendo la familia y la vida académica lo que más inquieta su salud mental; perdiendo el control algunas veces cuando sienten que su salud mental está siendo afectada. Por otro lado, aunque perciben la amenaza que representan las sustancias psicoactivas, el $30 \%$ de estudiantes no percibe la severidad de que el consumir sustancias psicoactivas puede generar adicción.

Respecto a la susceptibilidad percibida, los estudiantes aprecian que su estado de salud mental es el resultado de la estabilidad afectivo-emocional, los hábitos y su autoestima. Aproximadamente un $8 \%$ de la población ha presentado situaciones que afectan su salud mental. Así mismo, aunque se creen vulnerables, el reconocimiento de presentar situaciones que no permitan una buena salud mental es bajo, lo que es coherente con sus altos niveles de satisfacción respecto a su actual estado de salud mental.

Dentro de las barreras percibidas por los estudiantes para su salud mental se encuentra la facilidad para acceder a un profesional de la salud mental. Casi un $30 \%$ considera que nunca o casi nunca tiene facilidad para acceder a un profesional. Si bien es cierto que

.


ISSN 1794-9831

E-ISSN 2322-7028 Vol. 13 No. 1

Ene - Jun 2016

Cúcuta, Colombia los estudiantes consideran la necesidad de acudir al psicólogo, el poder hacerlo se convierte en la barrera más importante. Frente a las barreras percibidas es claro que tienen mayor peso las externas y contextuales que las internas e individuales.

Dentro de los procesos autorregulatorios de la motivación intrínseca de los estudiantes, se encuentra la capacidad de poder disminuir el riesgo a través de comportamientos saludables, con elementos que involucran el balance y potencial de la salud, encontrándose actividades internas y externas para el desarrollo de estilos de vida saludables.

En la categoría de claves para la acción se observa una percepción de vulnerabilidad, aspecto que debe ser tenido en cuenta para el diseño y ejecución de un programa de intervención. Es primordial reconocer el papel fundamental que juegan los medios de comunicación al ser asumidos como claves externas y herramientas para la salud mental, así como el ambiente como clave para mantener una salud mental adecuada.

\section{CONFLICTO DE INTERESES}

Los autores declaran no tener ningún conflicto de intereses. 


\section{REFERENCIAS BIBLIOGRÁFICAS}

1. Merani A. Diccionario de psicología. Tratados y Manuales. México: Grijalbo; 1986. p. 146.

2. Organización Mundial de la Salud (OMS). Plan de acción sobre salud mental 2013-2020. ISBN 978924350602 9. [Internet]. Ginebra: OMS; 2013 [consultado 9 de noviembre de 2014]. Disponible en: http://apps.who.int/iris/ bitstream/10665/97488/1/9789243506029_spa.pdf

3. Schönfeld P, Brailovskaia J, Bieda A, Xiao Chi Zhang, Margraf J. Efectos del estrés cotidiano en la salud mental positiva y negativa: mediación de la autoeficacia. International. Journal of Clinical and Health Psychology 2016; $1: 1-10$.

4. Díaz-Sotelo OD. Prevalencia de los Diagnósticos de Salud mental en Colombia: Análisis de los registros del sistema Integral de información de la Protección Social - SISPRO. Cuadernos Hispanooamericanos de Psicología 2015; 14(2):65-74.

5. Tortella-Feliu $M$, Baños R, Barrantes $N$, Botella $C$, Fernández-Aranda F, García-Campayo J, et al. Retos de la investigación psicológica en salud mental. Clínica y Salud [Internet]. 2016 [consultado 8 de marzo de 2016];27(1):37-43. Disponible en: http://dx.doi.org/10.1016/j.clysa.2016.02.001

6. Organización Mundial de la Salud (OMS). Carta de Ottawa para la Promoción de la Salud, Ottawa: OMS; 1986.

7. Bastías-Arriagada EM, Stiepovich-Bertoni J. Una revisión de los estilos de vida de estudiantes universitarios iberoamericanos. Cienc. enferm. [Internet]. 2014 [consultado 8 de marzo de 2016]; 20(2):93-101. Disponible en: http://www.scielo.cl/scielo.php?script=sci_arttext\&pid=S0717-95532014000200010\&lng=es. http:// dx.doi.org/10.4067/S0717-95532014000200010

8. Páez-Cala ML. Universidades saludables: los jóvenes y la salud. Revista Archivos de Medicina (Col) 2012; 12 (2): 201-220.

9. Yepes S. Universidad que promueve la salud: una propuesta para construir un reto posible hacia la formación integral de jóvenes universitarias. Revista Electrónica de Educación y Psicología 2007; 3(5): 1-13

10. Molerio O, Pedraza L, Herrera L. Universidades por la salud: experiencias desde la implementación de un centro de bienestar universitario. Revista Congreso Universidad [Internet]. 2015 [consultado 8 de marzo de 2016]; IV (1):45-57. Disponible en: http://www.congresouniversidad.cu/revista/index.php/congresouniversidad/article/ view/1107/555

11. Ortego-Maté MC, López-González S, Álvarez-Trigueros ML. La vulnerabilidad percibida. Open Curse Ware (Aula Virtual) [Internet]. España: Universidad de Cantabria; 2014 [consultado 4 de noviembre de 2014]. Disponible en: http://ocw.unican.es/ciencias-de-la-salud/ciencias-psicosociales-i/materiales/bloque-tematicoiv/tema-14.-la-adherencia-al-tratamiento-1/14.5.2.4-la-vulnerabilidad-percibida

12. PérezE, Bautista S. Susceptibilidad percibida de Diabetes Mellitus en adultos no diagnosticados. Revista Psicologia Cientifica.com [Internet]. 2008 [29 de agosto de 2014]; 17). Disponible en: http://www.psicologiacientifica.com/ diabetes-mellitus-susceptibilidad-percibida

13. Cabrera G, Tascón J, Lucumí D. Creencias en salud: historia, constructos y aportes del modelo. Rev. Fac. Nac. Salud Pública [Internet]. 2001 [consultado 29 agosto de 2014]; 19(1): 91-101. Disponible en: http://www. fundacionfes.org/attachments/185_Creencias\%20en\%20salud.pdf

14. Flórez L. Psicología social de la salud: promoción y prevención. Colombia: Manual Moderno; 2007.

15. Granados MC. Unidos fortaleciéndonos como una Universidad promotora de Salud. Los Diez primeros años del proceso. [Internet]. Bogotá: Pontificia Universidad Javeriana, 2013 [consultado 2 de febrero de 2016]. Disponible en: http://www.javeriana.edu.co/documents/245769/305029/Publicacion+conmemorativa.pdf/ be4b1643-7288-49bf-9674-3a12a22faccc

16. Ortiz M, Ortiz E. Psicología de la salud: Una clave para comprender el fenómeno de la adherencia terapéutica. Rev Méd [Internet]. 2007 [consultado 29 de agosto de 2014]; 135: 647-652. Disponible en: http://www.scielo. $\mathrm{cl} / \mathrm{pdf} / \mathrm{rmc} / \mathrm{v} 135 \mathrm{n} 5 /$ art $14 . \mathrm{pdf}$

17. Becerra-Heraud S. Universidades saludables: una apuesta a una formación integral del estudiante. Revista de Psicología [Internet]. 2013 [consultado 8 de marzo de 2016]; 31(2). Disponible en: http://www.scielo.org.pe/ scielo.php?pid=S0254-92472013000200006\&script=sci_arttext

18. León S, Medina S, Barriga A, Ballesteros I, Herrera. Psicología de la salud y calidad de vida. [Internet]. Barcelona: Editorial UOC; 2004 [consultado 3 de febrero de 2016]. Disponible en: http://books.google.com.co/ books?id=esM3UUnq9kcC\&pg=PA64\&lpg=PA64\&dq=susceptibilidad + percibida\&source=bl\&ots $=$ YClguR7F sz\&sig=m9icoPQczU813km94bBQJpPJEyw\&hl=es\&sa=X\&ei=iHlfVICdEcqqgwSCsISoDw\&ved=0CDEQ6 AEwAw\# $\mathrm{v}=$ onepage $\& \mathrm{q}=$ susceptibilidad\%20percibida\&f=false

19. Sarmiento M. A mental health Profile of Higher Education Students. Procedia - Social and Behavioral 
Sciences. [Internet]. 2015 [consultado 2 de febrero de 2016]; 191: 12-20. Disponible en: http://ac.els-cdn.com/ S1877042815028669/1-s2.0-S1877042815028669-main.pdf?_tid=dbc4ba7c-f104-11e5-bb6c-00000aab0f01\&a cdnat=1458744067_595d5aa4d4199d33e27732de3fccfba9

20. Ministerio de Protección Social. Colciencias. Pontificia Universidad Javeriana. Encuesta Nacional de Salud Mental 2015. [Internet]. Bogotá: Minsalud, Colciencias; 2015 [consultado 2 de febrero de 2016]. Disponible en: www.minsalud.gov.co/sites/rid/Lists/BibliotecaDigital/RIDE/DE/presentacion-encuesta-nacional-saludmental-2015.pdf

21. Páez R, Ubillos L, Pizarro P, León M. Modelos de creencias de salud y de la acción razonada aplicados al caso del SIDA. Rev. de Psicol Gral. y Aplic. 1994; 47(2): 141-149.

22. CivitciA. Perceived Stress and Life Satisfaction in College Students: Belonging and Extracurricular Participation as Moderators. Procedia - Social and Behavioral Sciences. [Internet]. 2015 [consultado 4 de marzo de 2016]; 205:271281. Disponible en: http://ac.els-cdn.com/S1877042815050958/1-s2.0-S1877042815050958-main.pdf? tid=643eca5c-f11c-11e5-8f1a-00000aab0f6c\&acdnat=1458754174_b10d10bbad10d9a643dc3af0daf70226

23. Rosales Y, Rosales F. Burnout estudiantil universitario. Conceptualización y estudio. Salud Ment 2013; 36(4): 337-345.

24. Giraldo A. La promoción de la salud como estrategia para el fomento de estilos de vida saludables. Revista Hacia la Promoción de la Salud 2010; 15(1):128-143.

25. Hunt J, Eisenberg D. Mental Health Problems and Help-Seeking Behavior Among College Students., Journal of Adolescent Health [Internet]. 2010 [consultado 4 de marzo de 2016]; 46:3-10. Disponible en: http://www. jahonline.org/article/S1054-139X(09)00340-1/pdf

26. Rodríguez J. Manual de psicología social de la salud. Madrid: Síntesis; 2008.

27. Ribeiro-Rodrigues ES, Cheik NC, Mayer AF. Nivel de atividad física e tabagismo em universitarios. Rev Saúde Pública 2008; 42(4): 672-678.

28. Zambrano-Plata GE, Toscano-Moros JP, Gil-Marles J. Actitudes sexuales en adolescentes estudiantes universitarios. Rev. cienc. cuidad. 2015; 12(1): 93-104.

29. Villamizar $D$, Mogollón O. Intervención en estudiantes universitarios: una experiencia investigativa y propositiva en relaciones de pareja. Pamplona: Editorial Universidad de Pamplona; 2014.

30. Ministerio de Salud y Protección Social. Plan decenal de salud pública 2012-2021. [Internet]. Bogotá: Minprotección; 2013 [consultado 9 de noviembre de 2014]. Disponible en: https://www.minsalud.gov.co/ Documentos\%20y\%20Publicaciones/Plan\%20Decenal\%20-\%20Documento\%20en\%20consulta\%20para\%20 aprobaci\%C3\%B3n.pdf 